\title{
Esperienze didattiche di modellizzazione emergente: uno sguardo ai processi di matematizzazione
}

\section{Educational experiences of emergent modelling: a look at the mathemati- zation processes}

\author{
Simone Passarella \\ Dipartimento di Matematica “Tullio Levi-Civita”, Università di Padova - Italia \\ 凶simone.passarella@unipd.it
}

Sunto / In questo contributo si presentano due esperienze didattiche di modellizzazione emergente. Dopo aver chiarito i costrutti di modellizzazione emergente e matematizzazione, vengono proposte due esperienze didattiche. La prima, condotta in una classe seconda della scuola primaria, ha riguardato la proprietà distributiva della moltiplicazione rispetto all'addizione; la seconda, in una classe quarta di una scuola secondaria di secondo grado, ha riguardato il calcolo del volume di solidi non noti. La descrizione di queste esperienze segue tre direzioni principali: l'esplicitazione dell'obiettivo di apprendimento; la scelta del problema nel contesto; l'analisi dei processi di matematizzazione orizzontale e verticale presenti nelle strategie risolutive degli studenti. Le esperienze descritte rappresentano un esempio significativo dell'importanza di proporre agli studenti attività di modellizzazione emergente, supportando gli studenti in un processo di reinvenzione di concetti matematici a partire dalle loro informali strategie risolutive.

Parole chiave: modellizzazione matematica; modellizzazione emergente; matematizzazione.
Abstract / In this contribution, two teaching experiences of emergent modelling are presented. After having clarified the constructs of emergent modelling and mathematization, two teaching experiences are proposed. The first one, carried out in a $2^{\text {nd }}$ grade class of a primary school, concerned the distributive property of multiplication over addition; the second one, carried out in a $12^{\text {th }}$ grade class of an upper secondary school, concerned the calculus of the volume of solids. The description of these experiences follows three main directions: the presentation of the learning objective; the choice of the problem in context; the analysis of horizontal and vertical mathematization processes in the students' solution strategies. The experiences described represent a significant example of the importance of proposing emergent modelling activities to students, supporting them in a process of re-invention of mathematical concepts starting from their informal solving strategies.

Keywords: mathematical modelling; emergent modelling; mathematization. 


\section{Introduzione}

Lo sviluppo della capacità di utilizzare la matematica per comprendere e risolvere problemi in situazioni reali è considerato in tutto il mondo come uno dei principali obiettivi dell'educazione matematica (Eurydice, 2011; National Council of Teachers of Mathematics [NCTM], 2000; Organization for Economic Co-operation and Development [OECD], 2004, 2006, 2013, 2016). In ambito italiano, tale capacità è sottolineata nelle stesse Indicazioni Nazionali per il Primo Ciclo di Istruzione (Ministero dell'Istruzione, dell'Università e della Ricerca [MIUR], 2012) e nelle Indicazioni Nazionali per i Licei (MIUR, 2010), nelle quali viene indicato come la conoscenza matematica debba fornire strumenti adatti a percepire, interpretare e collegare eventi della vita quotidiana. Lo studente, quindi, deve imparare ad analizzare situazioni reali traducendole in termini matematici e a scegliere opportune azioni da compiere in modo da produrre una soluzione efficace del problema. Tali competenze sono in linea con la strategia educativa in didattica della matematica della modellizzazione matematica (Kaiser, 2017). Modellizzare non significa solamente risolvere problemi reali, ma dar vita a un processo di matematizzazione e riflessione sulla matematica che porti alla costruzione di nuovi concetti e strumenti matematici (Passarella, 2021). Benché l'importanza della modellizzazione matematica sia ampiamente riconosciuta a livello nazionale e internazionale, nella pratica scolastica le attività di modellizzazione ricoprono ancora un ruolo marginale (Blum \& Borromeo Ferri, 2009), anche a causa di una resistenza da parte degli insegnanti ad abbandonare modelli didattici tradizionali e di tipo prevalentemente trasmissivo (MIUR, 2018). Gli stessi insegnanti richiedono infatti maggior supporto, formazione e materiali, quali ad esempio prototipi di pratiche didattiche, al fine di realizzare nelle loro classi attività significative di modellizzazione matematica (Bonotto \& Passarella, 2019).

Nel presente contributo si descrivono due esperienze didattiche di modellizzazione matematica nella prospettiva della modellizzazione emergente e con particolare attenzione ai processi di matematizzazione. Prima di presentare queste esperienze, si ripercorreranno i principali riferimenti teorici relativi alla modellizzazione e alla matematizzazione in didattica della matematica, al fine di chiarire e specificare il significato che questi termini ricoprono nel resto del lavoro.

In conclusione, di questa introduzione, si desidera specificare che in questo articolo con il termine modello si intende un sistema di strutture concettuali usate per interpretare e descrivere matematicamente una certa situazione (Richardson, 2004).

\section{Modellizzazione e matematizzazione}

\subsection{Modellizzazione matematica}

Storicamente lo sviluppo della modellizzazione matematica e del suo insegnamento è stato influenzato da due principali prospettive (Kaiser-Messner, 1986): la prospettiva pragmatica e quella scientifico-umanistica, le quali, benché condividano l'importanza di introdurre aspetti del mondo reale nell'insegnamento della matematica, presentano significative differenze. Secondo la prospettiva pragmatica, gli studenti dovrebbero imparare ad applicare la matematica per risolvere problemi del mondo reale (Pollak, 1968, 1969, 1979). Di conseguenza, la modellizzazione è vista come un processo ciclico dal mondo reale a quello della matematica e viceversa (Pollak, 1979), sottolineando I'interazione reciproca tra realtà e matematica applicata (Kaiser, 2017). La prospettiva scientifico-umanistica, invece, si sviluppa attorno a due direttrici principali: (i) la prima considera la matematica 
come una scienza, cioè una disciplina caratterizzata da strutture formali e non formali; (ii) la seconda si concentra sull'ideale educativo di enfatizzare l'abilità degli studenti nel creare relazioni tra la matematica ed il mondo reale. Di conseguenza, in questa prospettiva il fine della modellizzazione è quello di supportare gli studenti in processi di matematizzazione (si veda il par. 2.2). A partire da queste due prime prospettive sulla modellizzazione matematica, si sono sviluppati diversi approcci, differenziati per scopi, sfondi epistemologici, e relazioni rispetto alle prospettive iniziali (Kaiser \& Sriraman, 2006). Tra questi approcci ricordiamo quello della modellizzazione emergente (emergent modelling), nella quale gli elementi fondanti sono gli esempi dal mondo reale e le loro relazioni con la matematica stessa (De Lange, 1987; Freudenthal, 1991; Treffers, 1987). La modellizzazione emergente è stata teorizzata inizialmente da Gravemeijer (1999). La teoria di riferimento è quella della Realistic Mathematics Education (RME), nella quale i modelli rappresentano uno strumento per supportare un processo di re-invenzione (Freudenthal, 1991) della matematica da parte degli studenti. Infatti, attività di modellizzazione vengono qui usate come veicoli per re-inventare piuttosto che applicare concetti matematici (Greer et al., 2007). Gli studenti, partendo da situazioni reali, iniziano a modellizzare le proprie strategie matematiche informali, giungendo a re-inventare concetti matematici e applicazioni di cui hanno bisogno. Questi concetti sono successivamente formalizzati in termini matematici e generalizzati ad altre situazioni. II ruolo del modello, quindi, cambia durante il processo di modellizzazione, da specifico per la situazione reale di partenza a generale. La modellizzazione emergente è quindi un processo dinamico a lungo termine nel quale si realizza un passaggio da un modello delle strategie matematiche informali situate degli studenti a un modello per un ragionamento matematico formale e generale (Gravemeijer \& Doorman, 1999). Questo passaggio modello di-modello per ha come naturale conseguenza la costruzione di una nuova realtà matematica formale (Streefland, 1985), condivisa e significativa per gli studenti. Si ricorda infine come in Gravemeijer (2020) vengano descritti alcuni punti chiave per la progettazione e la realizzazione di attività di modellizzazione emergente, tra i quali l'identificazione dell'obiettivo di apprendimento (identify the learning goal) e l'identificazione di contesti di partenza (identify instructional starting points). Infatti, uno dei capisaldi della modellizzazione emergente è che il processo di modellizzazione ha inizio da un problema nel contesto che sia esperienzialmente significativo per gli studenti e ricco di stimoli matematici.

I diversi approcci alla modellizzazione matematica hanno dato vita a diverse connotazioni del processo di modellizzazione stesso, focalizzandosi maggiormente ora sulla soluzione del problema di partenza, ora sullo sviluppo di concetti matematici. Corrispondentemente, sono stati realizzati vari cicli di modellizzazione (per una panoramica dettagliata si veda Borromeo Ferri, 2006). II ciclo di modellizzazione è un ciclo ideale esplicitato tramite diverse fasi che descrivono il processo di modellizzazione per risolvere problemi reali (Kaiser, 2017). Esso non è solamente uno strumento teorico, ma può essere utilizzato dagli studenti nel processo di apprendimento e dall'insegnante come strumento diagnostico (Borromeo Ferri, 2018). Riportiamo in Figura 1 il ciclo di modellizzazione realizzato da Kaiser e Stender (2013), nel quale le caratteristiche fondamentali sono le seguenti:

- la situazione reale di partenza, dopo essere stata compresa, viene semplificata e strutturata per costruire un modello reale della situazione stessa, facendo assunzioni, formulando ipotesi, e identificando dati e fattori rilevanti;

- il modello reale viene tradotto in termini matematici in un modello matematico (matematizzazione);

- a partire dal modello matematico realizzato segue una fase di lavoro con la matematica stessa per trovare dei risultati matematici come soluzione del problema;

- i risultati matematici ottenuti vengono interpretati come risultati reali, i quali a loro volta devono essere validati rispetto al contesto inziale. Da qui eventualmente può aver inizio un nuovo ciclo, totale o parziale. 


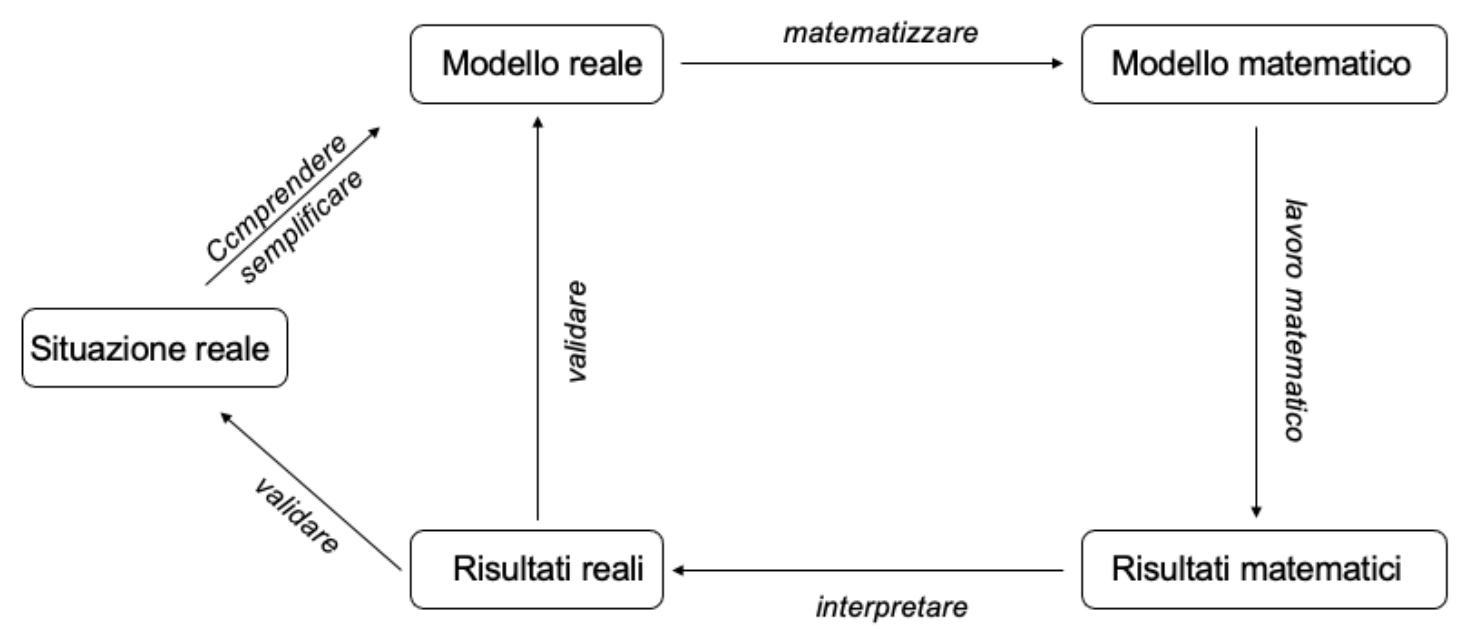

Figura 1. Ciclo di modellizzazione di Kaiser e Stender (2013)

\subsection{Matematizzazione}

Nel ciclo di modellizzazione riportato in Figura 1, il processo di matematizzazione viene considerato solo come una delle fasi che compongono l'intero processo di modellizzazione, e precisamente la fase di traduzione in termini matematici del modello reale della situazione di partenza. Tuttavia, in letteratura, con il termine matematizzazione ci si riferisce anche ad altri processi, che ricordiamo di seguito.

Una definizione di matematizzazione viene proposta da Jupri e Drijvers (2016), riferendosi a un'attività di organizzazione e analisi di una situazione reale con l'utilizzo di strumenti matematici. Tale definizione si rispecchia nel ciclo di matematizzazione (Figura 2) delineato in OECD (2004), ripreso e sottolineato in $\operatorname{OECD}(2013,2016)$, e descritto ampiamente in Franchini et al. (2017). Tale ciclo in effetti rievoca il ciclo di modellizzazione presentato nel paragrafo precedente, anche se con alcune differenze terminologiche:

- il punto di partenza è un problema reale, qui chiamato problema nel contesto. A partire da questa situazione problema reale, tramite un processo di formulazione si estrapolano le informazioni necessarie per tradurre il problema nel contesto in un problema matematico;

- strategie risolutive note o elaborate nel corso del processo stesso vengono utilizzate per risolvere il problema matematico, giungendo a dei risultati matematici del problema;

- le soluzioni matematiche dapprima vengono interpretate in funzione del contesto reale, ed infine valutate come accettabili o meno in base alle condizioni reali poste dal problema.

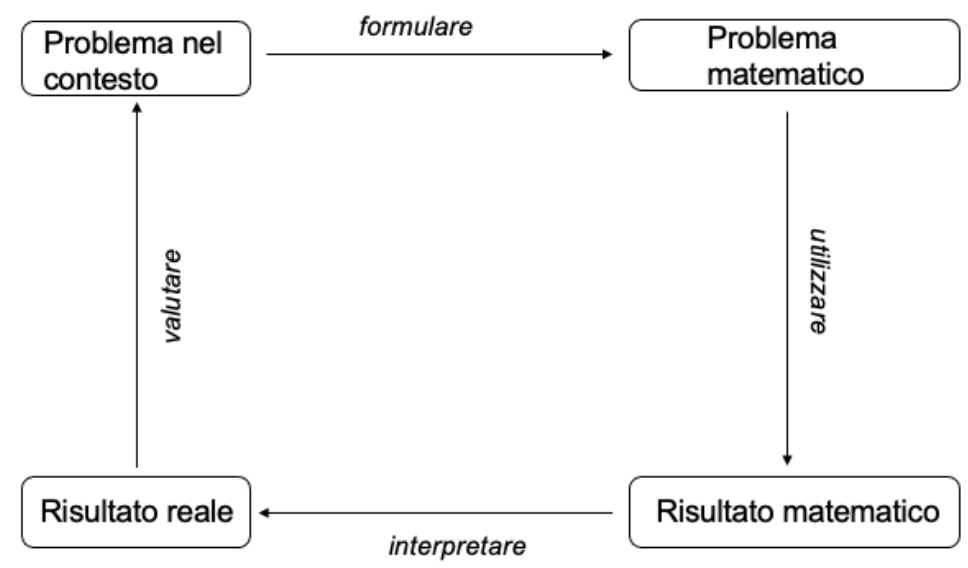

Figura 2. Ciclo di matematizzazione da OECD (2013). 
Il ciclo di matematizzazione può avere diverse funzioni. Ad esempio, Franchini et al. (2017) hanno utilizzato tale ciclo per analizzare possibili difficoltà degli studenti nella comprensione di quesiti dalle Prove Standardizzate di Matematica in Canton Ticino, mostrandone così le potenzialità come un utile strumento diagnostico e di suggerimento di possibili interventi futuri.

La definizione precedente di matematizzazione, e il ciclo in Figura 2, possono essere ulteriormente specificati in termini di processi matematici. Infatti, la teoria della RME, già citata in precedenza, riguardo alle relazioni reciproche tra matematica e mondo reale descrive due tipi di matematizzazione: matematizzazione orizzontale e matematizzazione verticale (Treffers, 1987). Nella matematizzazione orizzontale gli studenti usano strumenti e oggetti matematici per organizzare e risolvere problemi situati nel mondo reale, rispecchiando il movimento dal mondo reale alla matematica e viceversa. La matematizzazione verticale, invece, rappresenta un movimento interno a un mondo specifico; si riferisce cioè a processi che avvengono all'interno del mondo matematico quando occorre riconoscere relazioni tra oggetti interni alla matematica, mobilitando o eventualmente re-inventando nuovi concetti o strategie (Freudenthal, 1991), oppure all'interno del mondo reale quando un certo dato o una certa soluzione vanno validati nel contesto reale in cui si opera. In relazione ai cicli di modellizzazione e matematizzazione presentati, quindi, i processi di matematizzazione orizzontale e verticale si riferiscono a specifiche fasi di tali cicli (Tabella 1).

\begin{tabular}{|l|l|l|}
\hline & Ciclo di modellizzazione & Ciclo di matematizzazione \\
\hline $\begin{array}{l}\text { Matematizzazione } \\
\text { orizzontale }\end{array}$ & $\begin{array}{l}\text { Comprendere e semplificare } \\
\text { Matematizzare } \\
\text { Interpretare }\end{array}$ & $\begin{array}{l}\text { Formulare } \\
\text { Interpretare }\end{array}$ \\
\hline $\begin{array}{l}\text { Matematizzazione } \\
\text { verticale }\end{array}$ & $\begin{array}{l}\text { Lavoro matematico } \\
\text { Validare }\end{array}$ & $\begin{array}{l}\text { Utilizzare } \\
\text { Valutare }\end{array}$ \\
\hline
\end{tabular}

Tabella 1. Matematizzazione orizzontale e verticale nelle fasi del ciclo di modellizzazione e del ciclo di matematizzazione.

\subsection{Una visione di sintesi}

Nei paragrafi precedenti si sono presentati i principali riferimenti teorici relativi alla modellizzazione matematica e alla matematizzazione, mostrando punti di similitudine e differenze, e sottolineando quei processi di matematizzazione orizzontale e verticale all'interno dei cicli di modellizzazione e matematizzazione (Tabella 1). In questo paragrafo si vuole dare una sintesi dei concetti presentati in precedenza, con lo scopo di specificare in quale modo i termini utilizzati nel resto di questo contributo siano stati considerati. Nello specifico in questo lavoro si adotterà la prospettiva della modellizzazione emergente (Gravemeijer, 1999), la quale come descritto in precedenza considera il processo di modellizzazione matematica come un processo di costruzione, o meglio re-invenzione, di concetti, strumenti, strategie matematiche a partire da contesti reali informali. Congiuntamente, si considererà il ciclo di modellizzazione emergente proposto in Figura 3. In particolare, con matematizzazione qui si intendono le due componenti di matematizzazione orizzontale e verticale, anch'esse evidenziate in Figura 3. Si precisa come nello schema proposto si sia deciso di inglobare le fasi di validare e valutare nel processo di matematizzazione orizzontale, essendo in stretta relazione con la fase precedente di interpretazione. Nel ciclo di modellizzazione emergente, che rappresenta una sintesi terminologica di quanto visto in precedenza, il punto fondamentale è il processo di matematizzazione verticale, rappresentato dalla fase di lavoro matematico. In particolare, in questa fase l'enfasi sta nel processo di re-invenzione (Freudenthal, 1991) da parte degli studenti di concetti matematici. 


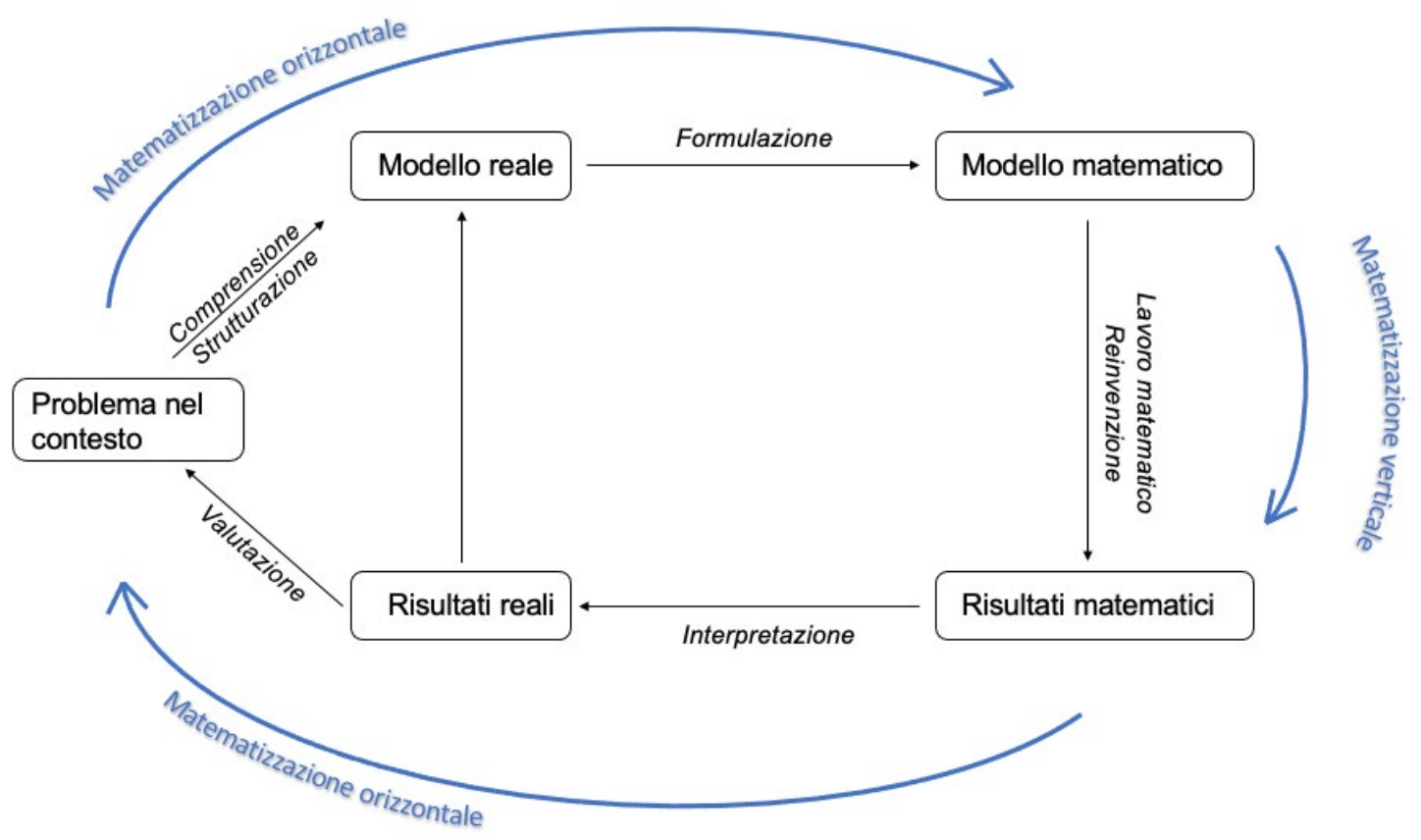

Figura 3. Ciclo di modellizzazione emergente.

Nelle esperienze presentate di seguito, al fine di identificare un contesto e problemi nel contesto per un'attività di modellizzazione emergente il cui scopo è quello di re-inventare un determinato concetto matematico, si adotterà l'euristica della fenomenologia didattica (didactical phenomenology) della RME. Secondo Freudenthal (1983), i concetti e oggetti matematici servono per organizzare fenomeni (phainomena), sia della vita reale che della matematica stessa (Bakker, 2004). La fenomenologia di un concetto matematico consiste nell'analisi di quel concetto in relazione ai fenomeni che organizza. Nello specifico, la fenomenologia didattica è lo studio di concetti (matematici) in relazione ai fenomeni con un interesse didattico. In questa prospettiva, la sfida è ricercare quei fenomeni che meglio sono organizzati dai concetti matematici che vogliamo che gli studenti apprendano, o meglio re-inventino. Nelle esperienze didattiche proposte l'individuazione di problemi nel contesto ha seguito una fenomenologia didattica, dove lo scopo è di trovare situazioni problema che possano rappresentare un punto di partenza per lo sviluppo di concetti o strumenti matematici che vogliamo che gli studenti sviluppino.

\section{Esempi di esperienze didattiche}

Si propongono di seguito due esperienze didattiche di modellizzazione emergente, realizzate una alla scuola primaria ${ }^{1}$ e l'altra alla secondaria di secondo grado. ${ }^{2}$ Tale scelta ha voluto mostrare come attività di modellizzazione emergente possano essere realizzate in diversi livelli scolastici, dalla scuola primaria alla secondaria di secondo grado, evidenziando come si possano attivare processi di matematizzazione orizzontale e verticale negli studenti. Entrambe le esperienze sono state progettate e realizzate dal ricercatore, autore di questo articolo, dopo un confronto con gli insegnanti titolari di

1. La scuola primaria in Italia dura cinque anni e corrisponde alla scuola elementare nel Canton Ticino.

2. La scuola secondaria di secondo grado in Italia dura cinque anni e corrisponde all'ultimo anno di scuola media e alla scuola media superiore o scuole professionali nel Canton Ticino. 
matematica. Gli stessi insegnanti hanno partecipato alla presentazione e alla gestione delle attività in classe rispondendo a eventuali dubbi o richieste di chiarimento degli studenti, e prendendo parte alla discussione finale. Le attività di modellizzazione emergente qui descritte sono state divise in tre fasi principali, che hanno richiesto per il loro svolgimento circa 8 ore suddivise in due settimane. Nella prima fase introduttiva, di circa 2 ore, agli studenti è stato dato il testo del problema che avrebbero dovuto risolvere, assieme ai relativi materiali. II ricercatore con l'aiuto dell'insegnante di matematica ha letto assieme agli studenti il testo e i vari materiali, assicurandosi che gli studenti avessero compreso cosa avrebbero dovuto fare nelle lezioni successive. La seconda fase ha previsto un lavoro di gruppo in cui gli studenti hanno realizzato i loro modelli per risolvere il problema che era stato loro posto. Questa fase, centrale nell'attività di modellizzazione, ha richiesto 4 ore per il suo svolgimento. II ricercatore e l'insegnante erano a disposizione per chiarire eventuali dubbi. L'ultima fase, di 2 ore, ha previsto la presentazione del proprio lavoro da parte di ogni gruppo alla classe. A questo è seguito un momento di discussione guidato dal ricercatore in cui si è fatto il punto sulle strategie proposte dagli studenti e sui concetti matematici emersi durante l'attività. Nell'esperienza realizzata alla scuola secondaria, infine, si è dedicato un momento finale alla riflessione individuale in forma scritta, in cui è stato chiesto agli studenti di ripercorrere l'intera attività svolta, di riportarne eventuali punti di forza o debolezza e dichiarare se avrebbero voluto ripetere o meno un'attività simile in futuro. Nella presentazione delle due esperienze didattiche, dopo aver esposto i rispettivi obiettivi di apprendimento e le situazioni problema, si porrà l'attenzione sui processi di matematizzazione orizzontale e verticale che tali situazioni hanno attivato negli studenti.

\subsection{Un'esperienza di modellizzazione emergente nella scuola primaria 3.1.1 Obiettivo di apprendimento}

La prima esperienza didattica che si propone è stata presentata in dettaglio in Passarella (2021). Qui il focus sarà sulle fasi di matematizzazione orizzontale e verticale attivate negli studenti. L'esperienza didattica in questione è stata realizzata in una classe seconda primaria composta da 18 alunni. Al momento dell'esperienza didattica la classe stava lavorando sulla moltiplicazione. Nello specifico, l'insegnante aveva introdotto da circa due settimane la moltiplicazione come somma ripetuta. Tale nozione era chiara agli studenti al momento dell'attività di modellizzazione, fatto rilevato con un test iniziale, che però esula dallo scopo di questo contributo. In particolare, gli studenti sapevano effettuare operazioni tra numeri naturali a una cifra e moltiplicazioni con multipli di 10. L'obiettivo di apprendimento per questa attività di modellizzazione emergente è stato identificato nella proprietà distributiva (nel seguito P. D.) della moltiplicazione rispetto all'addizione: $a \times(b+c)=a \times b+a \times c$, con $a, b, c$ numeri naturali. Si ricorda brevemente come la P. D. sia fondamentale in algebra, per lo studio dei polinomi e spazi vettoriali, e caratterizzi le operazioni tra numeri interi, e per tale motivo sia importante introdurla sin dai primi anni della scuola primaria (Maffia \& Mariotti, 2020).

\subsubsection{Problema nel contesto}

Partendo dalle conoscenze degli studenti sulla moltiplicazione tra numeri naturali, e seguendo l'approccio della fenomenologia didattica (si veda il par. 2.3), si è considerato uno specifico contesto e una serie di attività che guidassero gli studenti a re-inventare la P. D. A tal fine, si è deciso di creare un problema in cui gli studenti incontrassero la necessità di svolgere operazioni tra due numeri naturali, uno a una cifra e uno a due cifre. Infatti, se consideriamo ad esempio la moltiplicazione $25 \times 4$, dove il primo termine è un numero naturale a due cifre e il secondo a una cifra, un approccio per svolgere questa moltiplicazione potrebbe essere il seguente. Innanzitutto, 25 può essere scomposto come $20+5$. Poi, si può moltiplicare $20 \times 4$ e $5 \times 4$. Nella prima moltiplicazione il termine 20 è un multiplo di $10(20=2 \times 10)$, e gli studenti al momento dell'attività sapevano come svolgere moltiplicazioni di 
questo tipo. La seconda moltiplicazione, $5 \times 4$, è tra numeri naturali a una cifra. Quindi, riassumendo $25 \times 4=(20+5) \times 4=(20 \times 4)+(5 \times 4)$, che è proprio la P. D. Al fine di tradurre queste prime ipotesi in concrete attività in classe, si è scelto un contesto che fosse vicino alla realtà degli studenti e che allo stesso tempo prevedesse il fatto che nella sua risoluzione fosse necessario svolgere moltiplicazioni come quella descritta. Dato che al momento dell'attività la scuola era in ristrutturazione, si è deciso di creare il seguente problema:

II Direttore della scuola ha deciso di ristrutturare la scuola. Gli studenti potranno realizzare la piastrellazione del pavimento della loro aula. Il pavimento della vostra classe è stato diviso in sei strisce uguali. Ogni gruppo di studenti può piastrellare una di queste strisce utilizzando tutte le tipologie di piastrelle disponibili. Vi chiedo di creare un poster dove realizzate la piastrellazione della striscia assegnata al vostro gruppo, specificando il costo totale per la sua realizzazione e spiegando il procedimento che avete seguito.

Assieme al problema è stata data agli studenti una lista di piastrelle con il relativo costo (Figura 4), e con il vincolo di dover utilizzare tutte le tipologie per progettare la loro piastrellazione. In questo modo, si è pensato che una possibile strategia risolutiva potesse essere la seguente: (i) raggruppare e contare quante piastrelle dello stesso tipo erano state utilizzate; (ii) calcolare il costo associato a ogni tipo di piastrella utilizzato; (iii) sommare i costi relativi a ogni tipo di piastrella per ottenere il costo totale della piastrellazione realizzata. In particolare, dato che agli studenti è stato chiesto di piastrellare un'intera striscia della classe utilizzando tutte le tipologie di piastrelle disponibili, a un certo punto si suppone che il numero di piastrelle di uno stesso tipo sia dato da un numero naturale a due cifre, che dovrà essere moltiplicato per un numero naturale a una cifra dato dal costo relativo a quella tipologia di piastrella.

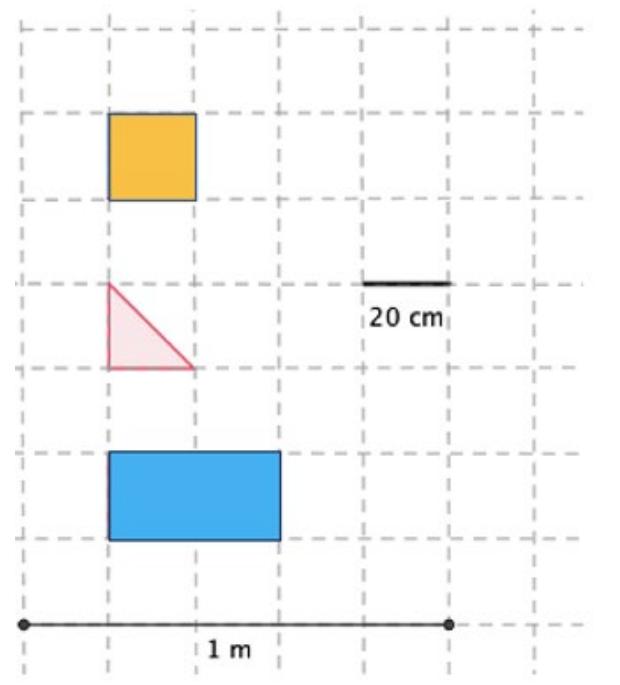

\begin{tabular}{|c|c|}
\hline piastrella & costo \\
\hline Quadrata & 6 euro \\
\hline Triangolare & 4 euro \\
\hline Rettangolare & 10 euro \\
\hline
\end{tabular}

Figura 4. Piastrelle che devono essere utilizzate dagli studenti e relativi costi.

\subsubsection{Processi di matematizzazione orizzontale e verticale}

Dopo un momento iniziale collettivo dedicato alla comprensione del problema, gli studenti sono stati divisi in sei gruppi dall'insegnante. Ad ogni gruppo è stato dato un poster con già disegnata in scala la striscia di cui avrebbero dovuto realizzare la piastrellazione (Figura 5). Si osservi che la striscia è stata suddivisa ulteriormente in quadrati $5 \times 5$, con l'idea di facilitare gli studenti inducendoli a concentrarsi su una porzione più piccola della striscia, la cui piastrellazione si sarebbe eventualmente potuta 
ripetere uguale. In realtà nessun gruppo ha ripetuto lo stesso schema, anzi in alcuni casi gli studenti hanno posizionato delle piastrelle rettangolari a cavallo della linea tratteggiata. In futuro, quindi, tale suddivisione potrebbe essere omessa.

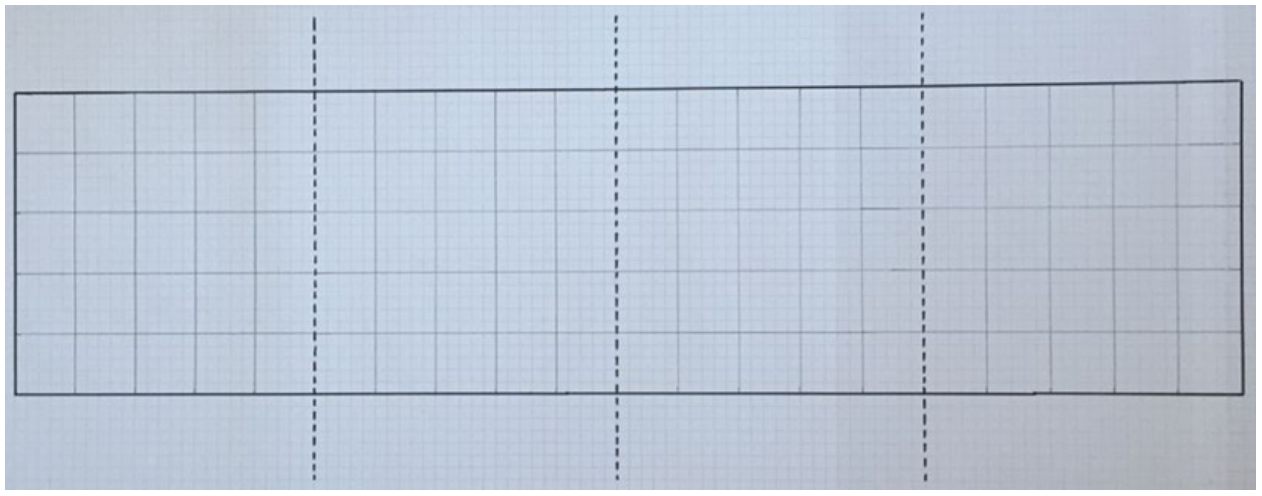

Figura 5. Poster dato a ogni gruppo di studenti

Gli studenti si sono approcciati al problema in modi differenti: chi prima ha realizzato uno schema con tutte le piastrelle, chi ha creato dei prototipi di piastrelle e le ha riportate nel poster (Figura 6).
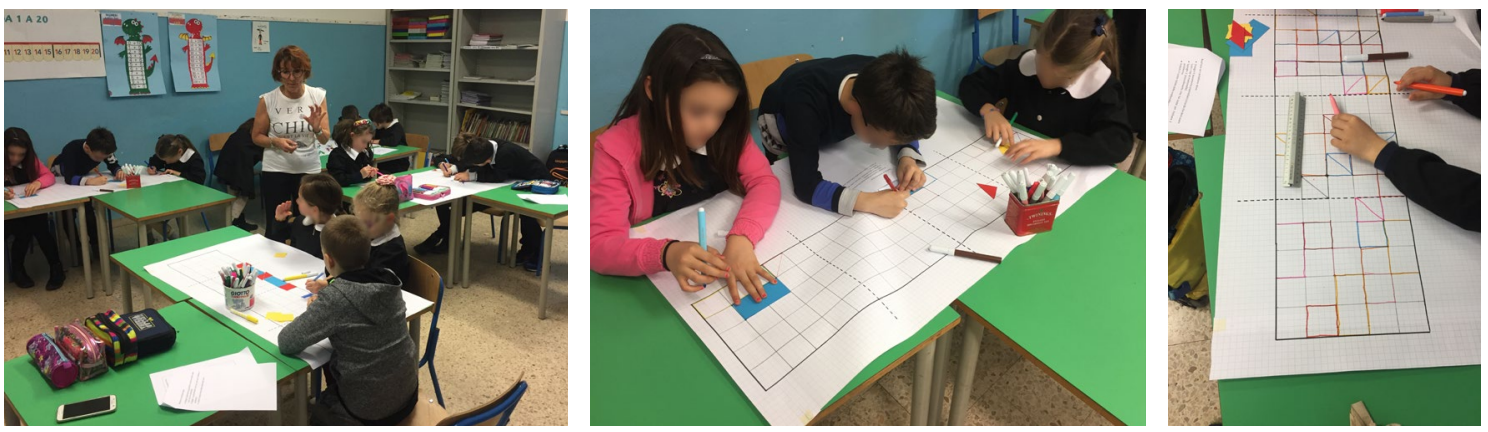

Figura 6. Studenti al lavoro.

Per risolvere il problema assegnato, gli studenti dei vari gruppi hanno sviluppato una strategia simile, e in accordo con le ipotesi formulate in fase di progettazione. Si riporta di seguito come esempio quanto realizzato da un gruppo. II primo step è stato quello di contare il numero di piastrelle dello stesso tipo. In questo esempio gli studenti hanno utilizzato 50 piastrelle quadrate, 26 triangolari e 15 rettangolari. Successivamente, il numero di piastrelle dello stesso tipo è stato moltiplicato per il relativo costo, cioè nel nostro esempio: $50 \times 6,26 \times 4,15 \times 10$. Si nota come gli studenti siano così giunti a dover eseguire moltiplicazioni in cui uno dei due termini è un numero naturale a due cifre. In due casi $(50 \times 6$ e $15 \times 10)$ tale termine è dato da un multiplo di 10 , che gli studenti già sapevano come trattare, ma nel restante caso $(26 \times 4)$ hanno dovuto sviluppare una nuova strategia. In particolare, uno degli studenti di questo gruppo ha suggerito di considerare il numero 26 come $20+6$, e successivamente moltiplicare $20 \times 4$ e $6 \times 4$, riconducendosi così a due moltiplicazioni note. Infine, sommare i due risultati ottenuti, giungendo quindi a una prima formulazione operativa informale della P. D. In Figura 7 è riportata la strategia adottata dagli studenti di questo gruppo. 


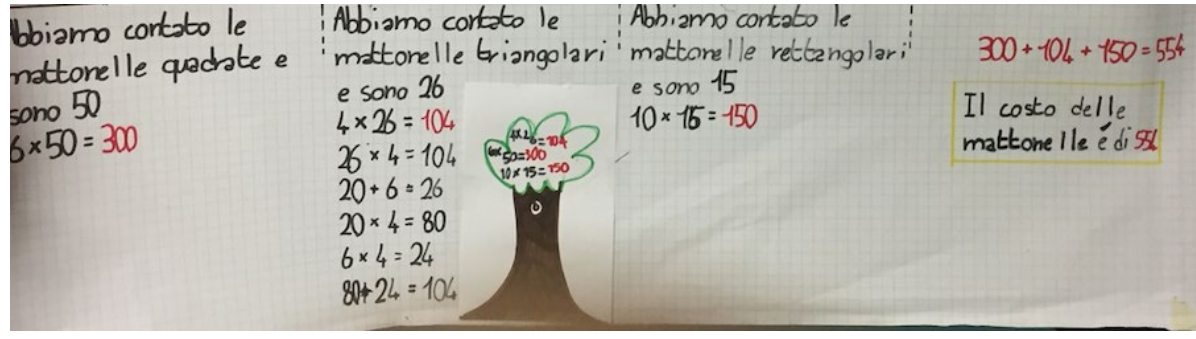

Figura 7. Strategia risolutiva realizzata da un gruppo di studenti.

Analizzando nel dettaglio la strategia sviluppata dagli studenti per risolvere il problema assegnato, si possono evidenziare chiaramente processi di matematizzazione sia orizzontale che verticale. Per quanto riguarda la matematizzazione orizzontale, questa è evidente inizialmente nelle fasi di comprensione-strutturazione del problema e di formulazione di un modello matematico. Dapprima gli studenti hanno realizzato un modello reale del problema, tramite una piastrellazione in termini di prototipi di piastrelle. Tale modello reale è stato realizzato dai diversi gruppi in maniera differente. Come mostrato in Figura 6, ad esempio, un gruppo ha costruito dei prototipi di piastrelle con dei cartoncini a forma di quadrato, triangolo e rettangolo. Tali prototipi sono stati utilizzati dagli studenti per ricoprire la striscia da piastrellare, riportandone il contorno. Dopo la realizzazione di un modello reale, che rappresenta la strutturazione del problema nel contesto di partenza, si è passati alla fase di formulazione di un modello matematico: i prototipi di piastrelle, infatti, sono stati interpretati in base alla loro forma, considerando quindi non più un pavimento piastrellato ma uno schema, o meglio un modello (matematico) costituito da diverse figure geometriche (quadrati, rettangoli e triangoli) che riempivano un rettangolo (la striscia). A questo punto ha inizio il processo di matematizzazione verticale, in cui gli studenti, lavorando con la matematica, hanno sviluppato una strategia risolutiva in due fasi: (i) contare le piastrelle dello stesso tipo, rappresentate da figure geometriche della stessa forma, e calcolarne il costo; (ii) sommare i risultati ottenuti per trovare il costo totale. In questa fase gli studenti sono stati in grado di re-inventare la P. D. a partire da una loro strategia risolutiva (la schematizzazione, ricostruita in fase di analisi, di tale strategia è presentata in Figura 8). Tuttavia, si può notare come la fase di interpretazione dei risultati matematici ottenuti non sia completa. Nell'esempio descritto, gli studenti danno come costo totale quello di 554, che manca dell'unità di misura. Gli studenti, quindi, arrivano a un risultato matematico che faticano a interpretare in maniera completa in relazione al contesto reale di partenza. In Figura 9 è rappresentato il ciclo di modellizzazione per l'esempio descritto, che mette in evidenza i processi critici come quello dell'interpretazione dei risultati matematici in relazione al contesto reale di partenza che, come detto, non risulta completo.

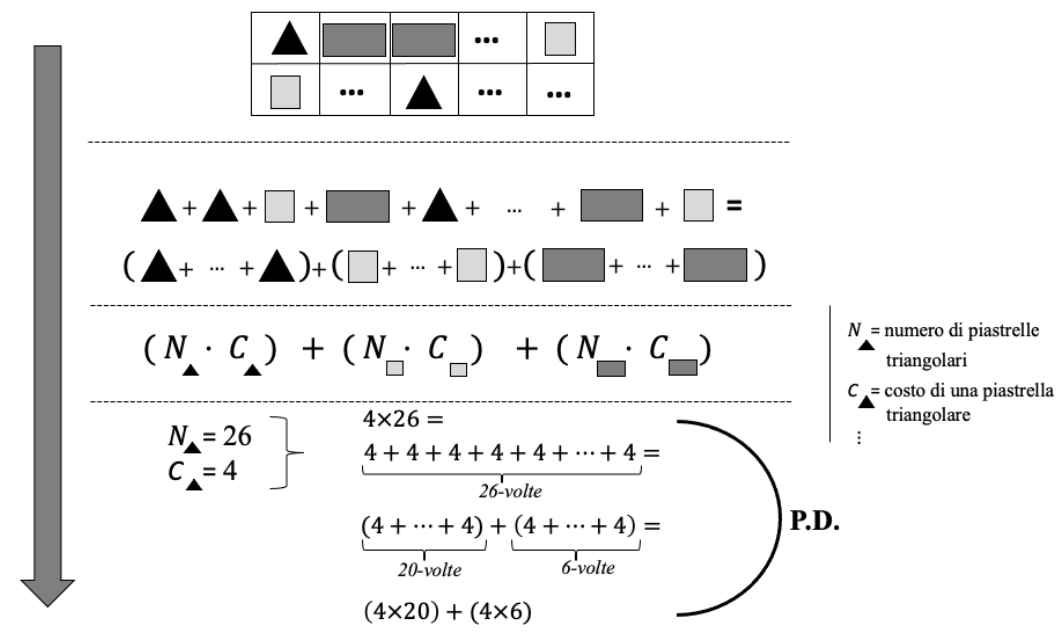

Figura 8. Processo di matematizzazione verticale. 


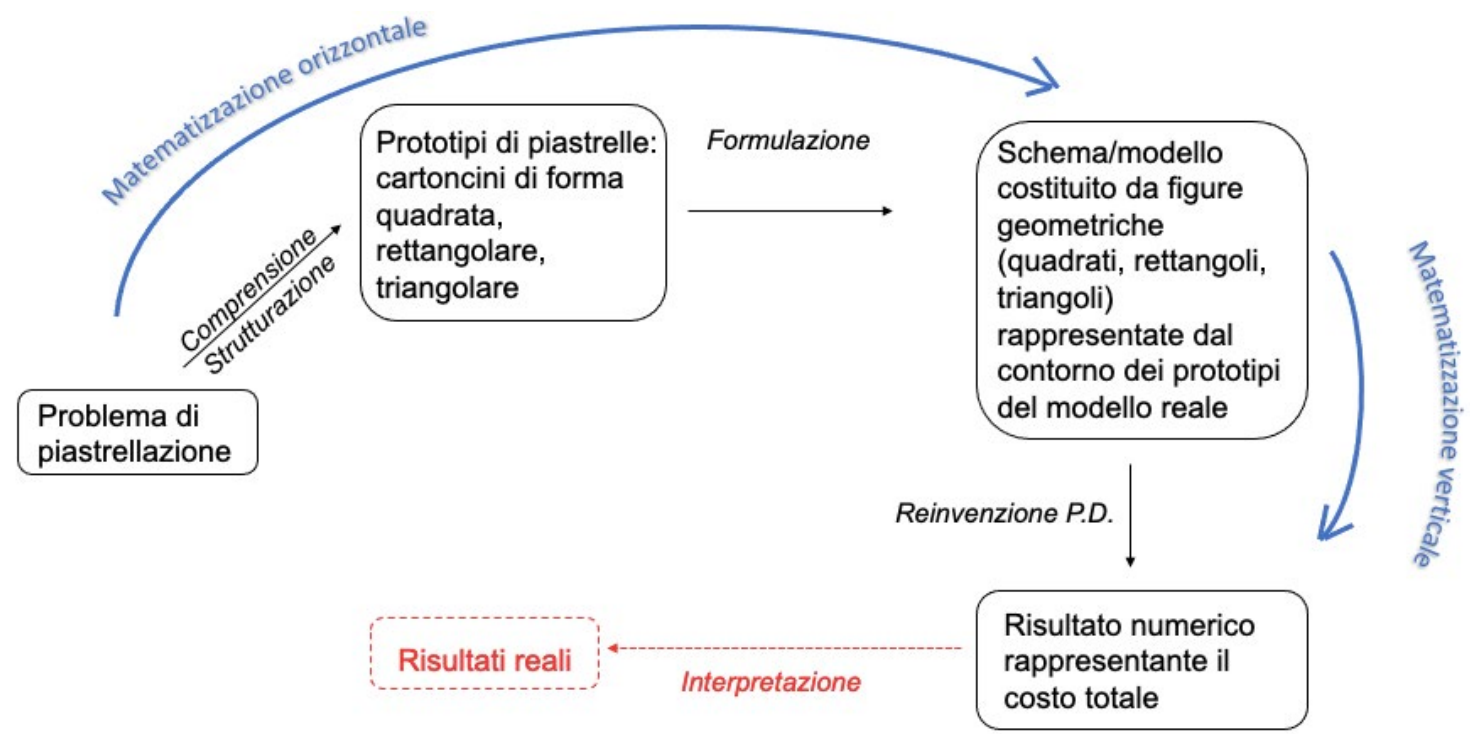

Figura 9. Ciclo di modellizzazione emergente relativo a una strategia utilizzata dagli studenti.

In conclusione, il contesto e il problema considerati hanno favorito la modellizzazione emergente, scandita al suo interno da processi di matematizzazione orizzontale e verticale. A partire dall'attività di modellizzazione proposta, I'insegnante può successivamente riprendere il concetto di P. D. sfruttando le strategie risolutive degli alunni, proponendo altri esempi e arrivando a una precisa formalizzazione di tale proprietà.

\subsection{Un'esperienza di modellizzazione emergente nella scuola secondaria di secondo grado 3.2.1 Obiettivo di apprendimento}

L'esperienza didattica di modellizzazione emergente di seguito riportata è stata realizzata in una classe quarta di una scuola secondaria di secondo grado (in particolare, Liceo Scientifico), composta da 25 studenti. Come argomento è stato scelto assieme all'insegnante di matematica di dedicarsi alla geometria euclidea tridimensionale, in linea con le Indicazioni Nazionali per il Liceo Scientifico (MIUR, 2010). Nello specifico, ci si è concentrati sul calcolo del volume di solidi come somma di volumi di solidi noti. Tale proprietà non è altro che la natura additiva della funzione volume, per la quale scomponendo un solido nell'unione di più solidi, il suo volume è la somma dei volumi di questi. Per semplicità ci si riferirà nel seguito a tale proprietà con il nome di principio di scomponibilità (abbreviato in P. S.), scelto come obiettivo di apprendimento per questa attività. Da quanto rilevato con un test iniziale, al momento dell'attività gli studenti non conoscevano tale principio, o almeno non lo sapevano utilizzare per calcolare il volume di solidi non noti.

\subsubsection{Problema nel contesto}

Secondo l'euristica della fenomenologia didattica, la domanda chiave è la seguente: quale problema può stimolare lo studente a re-inventare il P. S.? Nelle lezioni precedenti all'attività, I'insegnante di matematica aveva introdotto alcuni concetti di base di geometria euclidea dello spazio tramite alcune attività di gruppo con I'utilizzo di materiali quali cannucce, lattine, bottiglie. Conseguentemente, è stato proposto come problema per l'attività di modellizzazione quello riportato in Figura 10: 


\section{Bevandeitalia.srl}

Corso Stati Uniti, Padova

Oggetto: assunzione a tempo indeterminato addetto reparto imballaggi.

Bevandeitalia, leader mondiale nella produzione e distribuzione di bevande, ricerca addetto per reparto imballaggi. Contratto a tempo indeterminato di 19630 euro lordi annui. Per candidarsi è necessario presentare un progetto con allegato il proprio curriculum vitae. Il progetto richiede una stima del capitale necessario per la realizzazione di imballaggi per bevande, rispettando i seguenti vincoli:

- Litri iniziali da imballare di

\begin{tabular}{ccc}
\hline Acqua & Succo di frutta & Bibita gassata \\
\hline 1000 & 500 & 350 \\
\hline
\end{tabular}

- Diversificazione delle tipologie di imballaggio, secondo forma (almeno due per ogni bevanda) e materiali utilizzati.

Presentazione domanda: 18/01/19, presso Liceo Curiel, via Durer 14 Padova.

Figura 10. Problema per l'attività di modellizzazione emergente.

Assieme al testo del problema, agli studenti è stata fornita una brochure (Figura 11), in cui erano riportati alcuni materiali utili per la risoluzione del problema stesso. In questa brochure sono stati omessi alcuni dati, come la capacità delle bottiglie di vetro, al fine di incentivare il processo di re-invenzione del P. S. In questo modo gli studenti, per determinare la capacità delle bottiglie, avrebbero dovuto sviluppare una qualche strategia risolutiva che li avrebbe portati a riflettere sul concetto di scomponibilità. 


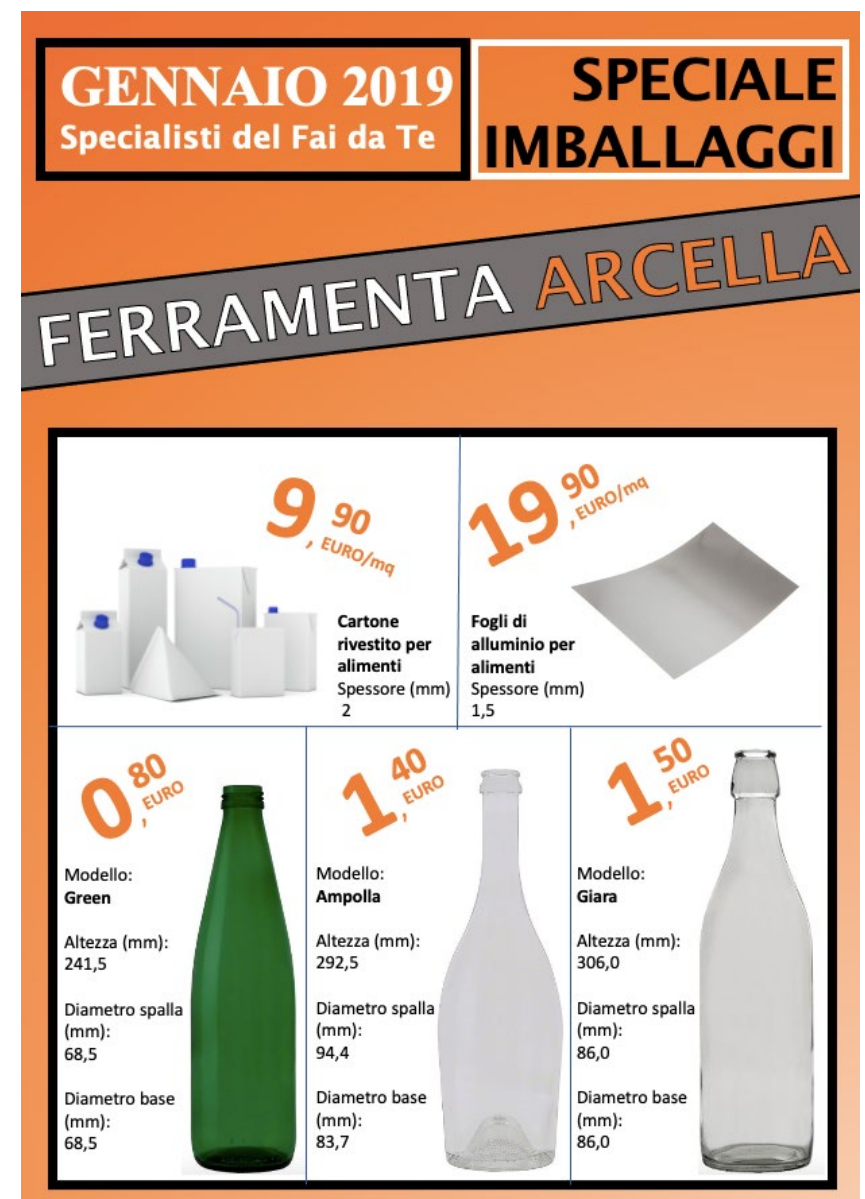

Figura 11. Brochure data agli studenti assieme al testo del problema.

\subsubsection{Processi di matematizzazione orizzontale e verticale}

Durante lo svolgimento del problema, gli studenti sono stati suddivisi in sei gruppi. Ogni gruppo è stato quindi invitato a presentare il progetto richiesto dal problema per candidarsi come addetto al reparto imballaggi. In particolare, ogni gruppo doveva realizzare almeno due tipologie di imballaggio per imballare 1000 litri di acqua, 500 litri di succo e 350 litri di bibita gassata. Al termine dell'attività ciascun gruppo ha dovuto presentare al resto della classe il proprio lavoro. Nella risoluzione del problema, alcuni gruppi di studenti hanno iniziato calcolando la capacità di diverse tipologie di imballaggi e scegliendo quelle la cui realizzazione fosse più economica. Altri, invece, si sono focalizzati su criteri di praticità, realizzando imballaggi per uso domestico o fuori casa. Tutti i gruppi, comunque, nei loro progetti finali, hanno incluso come tipologia di imballaggio per l'acqua diversi tipi di bottiglie tra quelle proposte nella brochure (Figura 11). Tutti i gruppi, in accordo con quanto ipotizzato nella progettazione dell'attività, hanno prima scomposto ogni bottiglia come somma di solidi noti di cui erano in grado di calcolare il volume, per poi sommare i volumi ottenuti e ricavare un'approssimazione del volume della bottiglia considerata, giungendo così a reinventare il P. S. Un esempio è riportato in Figura 12, dove la bottiglia modello green è stata scomposta come un cono più un cilindro, della stessa altezza. II volume totale ottenuto dalla somma dei volumi del cono e del cilindro era di 0,59 L, che è stato infine approssimato a 0,50 L. La giustificazione di tale approssimazione è dovuta, come riportano gli studenti, sia ad approssimazioni in fase di calcolo, sia al confronto del risultato ottenuto con i modelli già presenti sul mercato, sottolineando quindi l'attenzione dei ragazzi alla fase di valutazione dei risultati ottenuti in funzione del contesto reale del problema. 


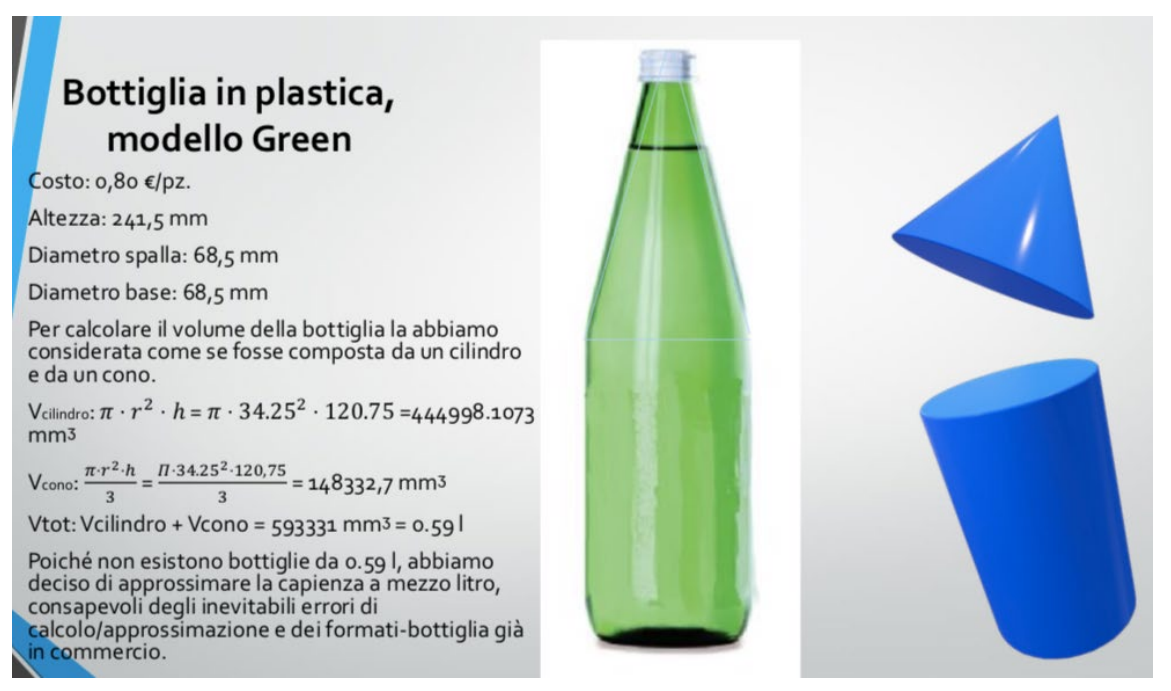

Figura 12. Strategia di un gruppo di ragazzi per calcolare la capacità della bottiglia green

Analizzando questo esempio, si possono evidenziare anche in questa strategia processi di matematizzazione orizzontale e verticale. Dapprima, secondo un processo di matematizzazione orizzontale, la situazione problema è stata strutturata, considerando diverse tipologie di bottiglie di vetro presenti nella brochure come tipologie di imballaggio adatte per l'acqua. Successivamente, gli studenti hanno formulato un modello matematico: ogni bottiglia può essere considerata come un solido non noto. Qui ha inizio un processo di matematizzazione verticale, nel quale studenti hanno sviluppato una strategia risolutiva che li ha portati a decomporre il solido rappresentante la bottiglia considerata in più solidi noti, riuscendone così a calcolare il volume, o meglio una sua approssimazione. Questo processo di matematizzazione verticale ha reso possibile la re-invenzione del P. S., supportando quindi il processo di modellizzazione emergente. Infine, gli studenti hanno previsto un ultimo processo di matematizzazione orizzontale, tramite l'interpretazione e valutazione del risultato ottenuto in termini del contesto reale di partenza. Questo è evidente, ad esempio, dall'approssimazione descritta in precedenza, dove gli studenti hanno tenuto in considerazione elementi della vita quotidiana, come la presenza sul mercato di bottiglie di acqua da mezzo litro. II ciclo di modellizzazione appena descritto è rappresentato in Figura 13.

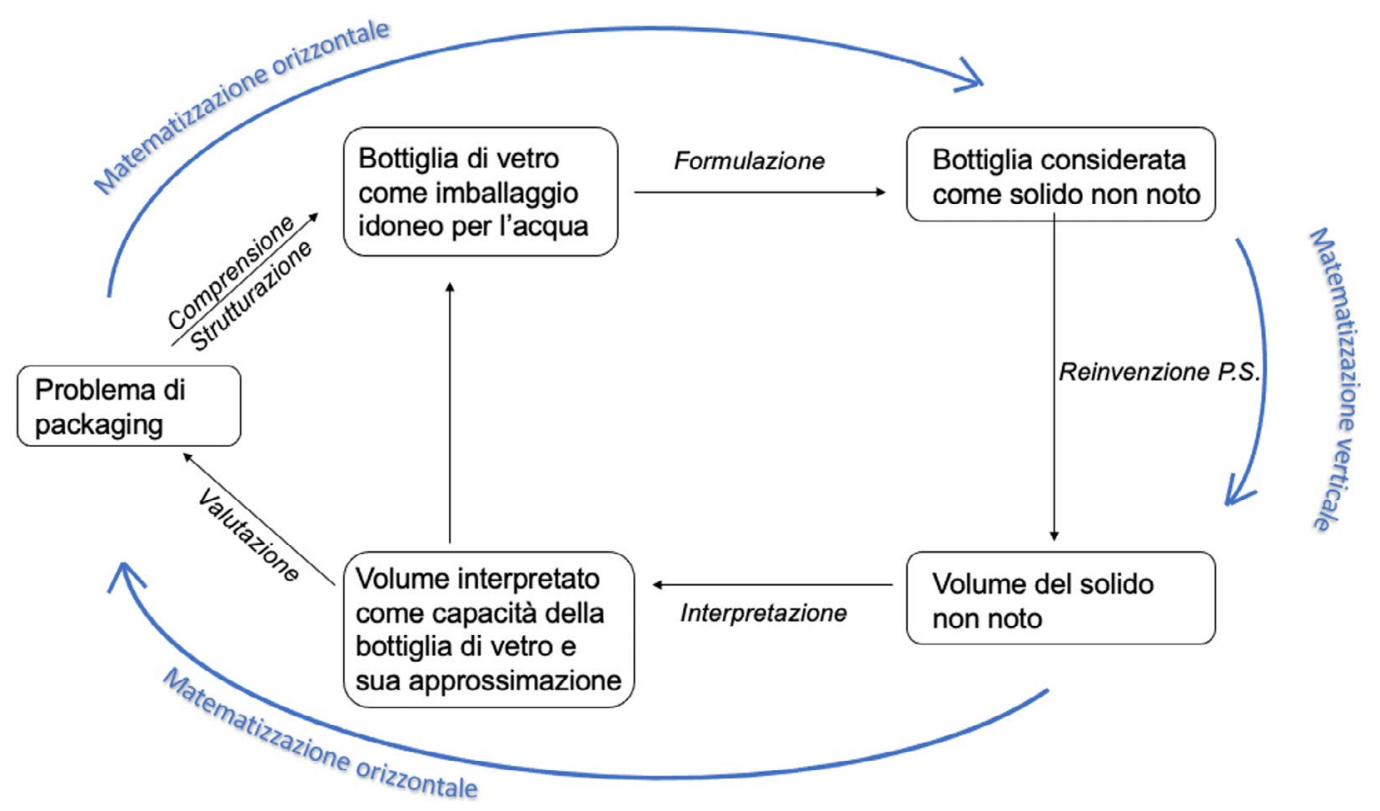

Figura 13. Ciclo di modellizzazione emergente relativo a una strategia utilizzata dagli studenti. 
Analizzando altri risultati di questa esperienza didattica, si può notare che in effetti i processi di matematizzazione orizzontale e verticale non sono sempre sequenziali come descritto in precedenza. Invece, essi risultano essere interconnessi durante tutto il ciclo di modellizzazione. Ad esempio, un gruppo di studenti nello scegliere alcuni modelli di imballaggio per imballare il quantitativo di bibita gassata, ha effettuato una ricerca storica che li ha portati a ragionare sulla presenza nel mercato di due tipologie di lattine, con la stessa capacità ma con costi di produzione differenti (Figura 14).

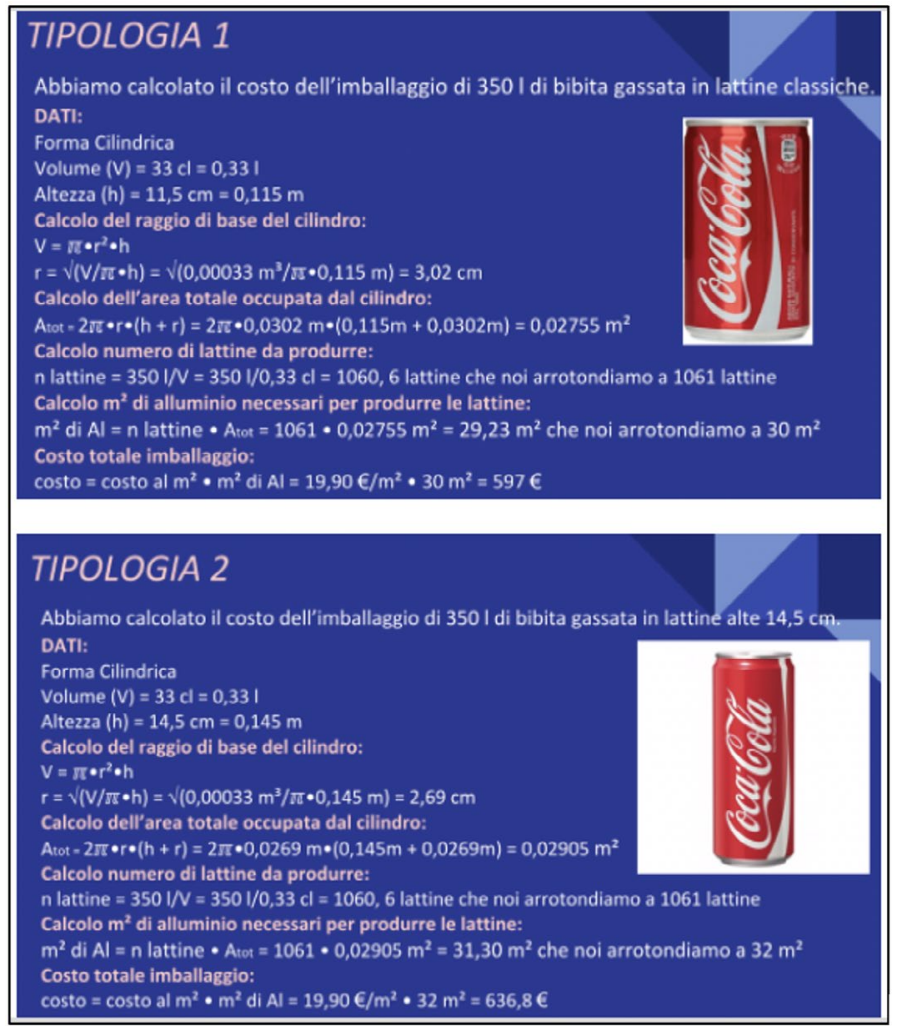

Figura 14. Confronto tra due tipologie di lattine presenti sul mercato.

Il processo di re-invenzione del P. S. attuato da tutti i gruppi e promosso dall'attività di modellizzazione emergente, come descritto nel caso del calcolo del volume delle bottiglie di vetro presenti nella brochure, è stato rilevato anche dagli studenti stessi. In alcune delle loro considerazioni riportate durante il momento finale di riflessione individuale, alcuni di loro hanno scritto:

«Nella realizzazione del progetto ho scoperto e usato il principio di scomponibilità».

«Per calcolare il volume delle bottiglie le abbiamo decomposte in solidi noti, usato proporzioni e formule per trovare i dati mancanti ed i volumi di questi solidi».

«ll lavoro è stato interessante e stimolante ed attraverso lo studio ed analisi degli imballaggi richiesti sono stato in grado di espandere la mia conoscenza matematica».

Si osservi che nella prima delle riflessioni riportate lo studente fa esplicito riferimento al P. S. Tale nome è stato introdotto dal ricercatore nella fase di presentazione dei gruppi, riferendosi alla strategia risolutiva sviluppata dagli studenti di calcolare il volume di un solido composto come somma di volumi di solidi noti. 


\section{Conclusioni}

Il presente contributo si è concentrato su alcune esperienze didattiche di modellizzazione matematica. Nella prima parte del contributo si sono ricordati i più significativi costrutti teorici relativi alla modellizzazione matematica e alla matematizzazione. Un'attenzione particolare è stata data al costrutto della modellizzazione emergente (Gravemeijer, 1999), cioè a quel processo in cui gli studenti, partendo da situazioni reali, iniziano a modellizzare le proprie strategie matematiche informali, giungendo a re-inventare concetti matematici e applicazioni di cui hanno bisogno. Parallelamente si è discusso il concetto di matematizzazione, con le sue diverse accezioni all'interno del ciclo di modellizzazione stesso e alle sue componenti di matematizzazione orizzontale e verticale (Treffers, 1987). A partire dai riferimenti teorici, si è proposta una sintesi, al fine di chiarire l'accezione data ai costrutti utilizzati in questo contributo. In particolare, in Figura 3 si è proposto il ciclo di modellizzazione emergente con particolare attenzione ai processi di matematizzazione orizzontale e verticale che lo caratterizzano. La seconda parte del contributo si è concentrata nel presentare alcune esperienze didattiche di modellizzazione emergente. La prima esperienza proposta è stata condotta in una classe seconda primaria e ha riguardato la proprietà distributiva della moltiplicazione rispetto all'addizione. La seconda esperienza didattica presentata è stata condotta in una classe quarta di una scuola secondaria di secondo grado e ha riguardato il principio di scomponibilità. In particolare, nel riportare queste esperienze ci si è concentrati su tre punti chiave per un'attività di modellizzazione, in accordo con Gravemeijer (2020): I'individuazione dell'obiettivo di apprendimento; I'individuazione del problema nel contesto; i processi di matematizzazione orizzontale e verticale nelle strategie risolutive degli studenti. In entrambe le esperienze, le proposte hanno permesso agli studenti di re-inventare alcuni concetti matematici (proprietà distributiva e principio di scomponibilità) a partire dalla risoluzione di un problema situato in un contesto reale che li ha stimolati ad attivare processi di matematizzazione orizzontale e verticale. Come evidenziato in particolare nella seconda esperienza proposta, tali processi non sono necessariamente consecutivi (matematizzazione orizzontale $\rightarrow$ matematizzazione verticale $\rightarrow$ matematizzazione orizzontale), ma possono anche essere intrecciati tra loro durante la risoluzione di un problema di modellizzazione.

Nelle esperienze descritte, i problemi proposti agli studenti, assieme ai materiali e vincoli forniti, hanno promosso il processo di re-invenzione auspicato in fase di progettazione. Tuttavia, si vuole prestare attenzione all'eventualità che le attività progettate non portino alla re-invenzione del concetto target, o che non tutte le soluzioni degli studenti conducano a tale re-invenzione. In quest'ultimo caso, un ruolo fondamentale è svolto dal docente nella discussione collettiva al termine dell'attività. Dopo che gli studenti hanno presentato i loro progetti, è bene quindi che si dedichi del tempo al confronto delle varie strategie risolutive, riflettendo su di esse e confrontandole, ed eventualmente sottolineando i concetti matematici che si volevano far emergere dall'attività di modellizzazione. Questo aspetto non è stato sviluppato in questo articolo, il cui scopo, si ricorda, era quello di analizzare i processi di matematizzazione orizzontale e verticale emersi nelle strategie risolutive ideate autonomamente dagli studenti nelle due esperienze di modellizzazione emergente. II ruolo dell'insegnante può rappresentare una posizione centrale nel favorire processi di matematizzazione sia orizzontale che verticale, e per tale motivo si ritiene necessario realizzare degli studi di caso in cui il ruolo dell'insegnante sia delineato chiaramente, in una prospettiva di re-invenzione guidata (Freudenthal, 1991) della matematica. Inoltre, risulta fondamentale riflettere sulla fase iniziale di progettazione. In particolare, nel caso in cui gli studenti non dovessero giungere alla re-invenzione dei concetti ipotizzati, si potrebbe scegliere di utilizzare un problema più focalizzato o aggiungere un vincolo al problema. Si ritiene quindi che un aspetto fondamentale sia rappresentato dalla fase di progettazione di un'attività di modellizzazione emergente, che in effetti è una fase fondamentale per la realizzazione di una qualsiasi attività 
didattica. Questo è in linea con la ricerca in didattica della matematica relativa al task design (Watson \& Ohtani, 2015). In questa prospettiva, infatti, la progettazione di un'attività non consiste solo nel creare problemi, ma è un processo complesso, che inizia con l'individuazione di uno o più obiettivi di apprendimento, l'individuazione di contesti e problemi che permettano il raggiungimento di tali obiettivi; la realizzazione di materiali e attività didattiche che concretizzino le precedenti ipotesi; la riflessione su quanto avvenuto effettivamente in classe.

In conclusione, le esperienze descritte rappresentano un esempio significativo dell'importanza di proporre agli studenti attività di modellizzazione emergente. Tali attività, infatti, possono supportare gli studenti in un processo di re-invenzione (Freudenthal, 1991) di concetti matematici a partire dalle loro informali strategie risolutive, come evidenziato nei due esempi riportati, in cui gli studenti sono giunti a re-inventare da un lato la proprietà distributiva della moltiplicazione rispetto all'addizione, dall'altro il principio di scomponibilità per il calcolo di volumi di solidi non noti. In questo modo gli studenti danno senso al loro fare matematica, poiché ancorato a un'attività per loro esperienzialmente significativa. A partire dalle strategie risolutive degli studenti, i concetti matematici emersi possono essere successivamente generalizzati ad altre situazioni, giungendo a costituire una realtà matematica formale significativa per lo studente.

\section{Bibliografia}

Bakker, A. (2004). Design research in statistic education: on symbolizing and computer tools. CD-Bèta Press.

Blum, W., \& Borromeo Ferri, R. (2009). Mathematical Modelling: Can It Be Taught And Learnt? Journal of Mathematical Modelling and Application, 1(1), 45-58.

Bonotto, C., \& Passarella, S. (2019). Modellizzazione e problem-posing come strumenti e traguardi dell'educazione matematica. L'insegnamento della matematica e delle scienze integrate, 42A(4), 469-488.

Borromeo Ferri, R. (2006). Theoretical and empirical differentiations of phases in the modelling process. ZDMThe International Journal on Mathematics Education, 38(2), 86-95.

Borromeo Ferri, R. (2018). Learning How to Teach Mathematical Modeling in School and Teacher Education. Springer.

De Lange, J. (1987). Mathematics, insight and meaning. OW\&OC, Rijksuniversiteit Utrecht.

Eurydice. (2011). Mathematics education in Europe: Common challenges and national policies. Education, Audiovisual and Culture Executive Agency.

Franchini, E., Lemmo, A., \& Sbaragli, S. (2017). II ruolo della comprensione del testo nel processo di matematizzazione e modellizzazione. Didattica della matematica. Dalla ricerca alle pratiche d'aula, 1, 38-63.

Freudenthal, H. (1983). Didactical phenomenology of mathematical structures. Reidel.

Freudenthal, H. (1991). Revisiting mathematics education. China lectures. Kluwer.

Gravemeijer, K. (1999). How emergent models may foster the construction of formal mathematics. Mathematical Thinking and Learning, 1(2), 155-177.

Gravemeijer, K. (2020). Emergent Modeling: an RME Design Heuristic Elaborated in a Series of Examples. Educational Designer, 4(13), 1-31. 
Gravemeijer, K., \& Doorman, M. (1999). Context Problems in Realistic Mathematics Education: A Calculus Course as an Example, Educational Studies in Mathematics, 39(1-3), 111-129.

Greer, B., Verschaffel, L., \& Mukhopadhyay. (2007). Modelling for life: mathematics and children's experience. In W. Blum, P. L. Galbraith, H.-W. Henn \& M. Niss (Eds.), Modelling and applications in mathematics education (ICMI 14th study) (pp. 89-98). Springer.

Jupri, A., \& Drijvers, P. H. M. (2016). Student difficulties in mathematizing word problems in algebra. EURASIA Journal of Mathematics, Science and Technology Education, 12(9), 2481-2502.

Kaiser, G. (2017). The Teaching and Learning of Mathematical Modeling. In J. Cai (Ed.), Compendium for Research in Mathematics Education (pp. 267-291). National Council of Teachers of Mathematics.

Kaiser, G., \& Sriraman, B. (2006). A global survey of international perspectives on modelling in mathematics education. ZDM-The International Journal on Mathematics Education, 38(3), 302-310.

Kaiser, G., \& Stender, P. (2013). Complex modelling problems in a co-operative, self-directed learning environment. In G. Stillman, W. Blum, J. Brown \& G. Kaiser (Eds.), Teaching mathematical modelling: Connecting to research and practice (pp. 277-293). Springer.

Kaiser-Messner, G. (1986). Anwendungen im Mathematikunterricht. Vol.1-Theoretische Konzeptionen. Vol.2-Empirische Unteruschungen. Franzbecker.

Maffia, A., \& Mariotti, A. (2020). From action to symbols: giving meaning to the symbolic representation of the distributive law in primary school. Educational Studies in Mathematics, 104, 25-40.

Ministero dell'Istruzione, dell'Università e della Ricerca. (2010). Indicazioni Nazionali per Licei. https://www.indi re.it/lucabas/lkmw file/licei2010/indicazioni nuovo impaginato/ decreto indicazioni nazionali.pdf

Ministero dell'Istruzione, dell'Università e della Ricerca. (2012). Indicazioni Nazionali per il Curricolo della Scuola dell'Infanzia e del Primo Ciclo d'Istruzione. http://www.indicazioninazionali.it/wp-content/uploads/2018/08/ Indicazioni Annali Definitivo.pdf

Ministero dell'Istruzione, dell'Università e della Ricerca. (2018). Indicazioni Nazionali e Nuovi Scenari. Documento a cura del Comitato Scientifico Nazionale per le Indicazioni Nazionali per il curricolo della scuola dell'infanzia e del primo ciclo di istruzione. https://www.miur.gov.it/documents/20182/0/Indicazioni+naziona li+e+nuovi+scenaril

National Council of Teachers of Mathematics. (2000). Principle and Standard for School Mathematics. NCTM.

Organization for Economic Co-operation and Development. (2004). The PISA 2003 Assessment Framework: Mathematics, Reading, Science and Problem Solving Knowledge and Skills. OECD Publishing.

Organization for Economic Co-operation and Development. (2006). Assessing Scientific, Reading and Mathematical Literacy: A Framework for PISA 2006. OECD Publishing.

Organization for Economic Co-operation and Development. (2013). PISA 2012 Assessment and Analytical Framework: Mathematics, Reading, Science, Problem Solving and Financial Literacy. OECD Publishing.

Organization for Economic Co-operation and Development. (2016). PISA 2015 Assessment and Analytical Framework: Science, Reading, Mathematics and Financial Literacy. OECD Publishing.

Passarella, S. (2021). Emergent modelling to introduce the distributivity property of multiplication: a design research study in a primary school. International Journal of Mathematical Education in Science and Technology. https://doi.org/10.1080/0020739X.2021.1910869

Pollak, H. O. (1968). On some of the problems of teaching applications of mathematics. Educational Studies in Mathematics, 1(1/2), 24-30. 
Pollak, H. O. (1969). How can we teach applications of mathematics? Educational Studies in Mathematics, 2, 393-404.

Pollak, H. O. (1979). The interaction between mathematics and other school subjects. In UNESCO (Ed.), New Trends in mathematics teaching IV (pp. 241-248). UNESCO.

Richardson, K. (2004). A design of useful implementation principles for the development, diffusion, and appropriation of knowledge in mathematics classrooms. Unpublished doctoral dissertation, Purdue University.

Streefland, L. (1985). Wiskunde als activiteit en de realiteit als bron (Mathematics as an activity and the reality as a source). Tijdschrift voor Nederlands Wiskundeonderwijs (Nieuwe Wiskrant), 5(1), 60-67.

Treffers, A. (1987). Three dimensions. A model of goal and theory description in mathematics instruction-the Wiskobas project. Reidel Publishing.

Watson, A., \& Ohtani, M. (2015). Task Design in Mathematics Education: An ICMI study 22. Springer. 